\title{
Necropolítica e a corrida tecnológica: notas sobre ensaios clínicos com vacinas contra o coronavírus no Brasil
}

Necropolitics and technological race: notes on clinical trials with coronavirus vaccines in Brazil

\author{
Rosana Castro' \\ https://orcid.org/0000-0002-1069-4785 \\ rosana.rc.castro@gmail.com \\ I Pesquisadora independente - Brasília, DF, Brasil
}




\title{
Resumo
}

Com o avanço da pandemia de Covid-19, iniciou-se uma corrida tecnológica para o desenvolvimento de vacinas. Diversas pesquisas estão em curso no mundo e quatro candidatas a vacina estão em teste no Brasil. Neste artigo, problematizo como dispositivos necropolíticos sanitários, econômicos e políticos, racial e socialmente articulados, configuraram o Brasil como um epicentro da pandemia e um "laboratório" de vacinas no ano de 2020, e analiso como essa situação foi localmente positivada como um reconhecimento internacional das competências do país em participar do mercado global de pesquisas farmacêuticas. Reflito, ainda, sobre como o envolvimento nos estudos não necessariamente garante o acesso futuro da população brasileira às vacinas aqui testadas, pois tais tecnologias são objeto de correntes disputas comerciais desiguais, que tendem a reforçar as condições que contribuíram para conduzir o país à tragédia sanitária da qual se espera surgir a solução para a pandemia.

Palavras-chave: Covid-19; ensaios clínicos; vacinas; necropolítica.

\begin{abstract}
With the advance of the COVID-19 pandemic, a technological race for vaccine development began. Several researches are under development in the world and four vaccine candidates are being tested in Brazil. In this article I discuss how racial and socially articulated sanitary, economic and political dispositifs configured Brazil as an epicenter of the pandemic and a vaccine "laboratory" in 2020. I analyze how this situation was positively absorbed as an international recognition of the country's competences participating in the global pharmaceutical research market. I also reflect on how the involvement in such studies does not necessarily guarantee Brazilian population's future access to the vaccines tested here, since these technologies are subject of current unequal commercial disputes, which tend to reinforce the conditions that contributed to lead the country to the health tragedy from which the solution to the pandemic is expected to emerge.
\end{abstract}

Keywords: COVID-19; clinical trials; vaccines; necropolitics. 


\section{Introdução ${ }^{1}$}

Com o rápido avanço da pandemia de Covid-19 nos primeiros meses de 2020, iniciou-se uma corrida tecnológica para desenvolvimento de vacinas contra o novo coronavírus. Sob imperativos de cientificidade, celeridade e competitividade, laboratórios privados, governos nacionais e consórcios multilaterais investiram cifras monumentais na produção de tecnologias concorrentes ao pioneirismo na imunização. Segundo a Organização Mundial da Saúde (OMS), até o final de agosto de 2020 havia 176 candidatas a vacinas em fase de pesquisas, das quais 33 estavam em fase de estudos clínicos, ou seja, experimentação em humanos (World Health Organization, 2020a). ${ }^{2}$ As iniciativas em curso incluem investimentos de parcerias entre órgãos públicos e privados, farmacêuticas multinacionais e consórcios multilaterais mediados por organismos internacionais. Quatro compostos candidatos à vacina começaram a ser testados no Brasil no primeiro semestre de 2020, situação que destacou o país na mídia internacional como um "laboratório de vacinas" (Andreoni; Londoño, 2020), de onde poderiam emergir evidências que atestassem a eficácia de vacinas com potencial de dar fim à pandemia. ${ }^{3}$

O destaque brasileiro no campo experimental está assentado, principalmente, no fato de aqui haver, durante o período de testes de vacinas e nos meses subsequentes, índices persistentemente altos de contágios e óbitos por Covid-19. Segundo registros da OMS, até meados de 2020 o Brasil foi o segundo país do mundo com a maior quantidade de casos e mortes pelo coronavírus, totalizando, até o final de agosto, aproximadamente 3,8 milhões de

1 Uma primeira versão deste artigo foi apresentada no “2o Webinar População negra em contexto de pandemia", realizado em modalidade on-line em julho de 2020 e organizado pelo Comitê de Antropólogas/os Negras/os da Associação Brasileira de Antropologia. Agradeço aos colegas do evento, coordenado por Ana Paula da Silva, e a seus participantes, bem como aos pareceristas anônimos pelos apontamentos que contribuíram para o aprimoramento do trabalho.

2 A quantidade de estudos clínicos saltou no segundo semestre de 2020 e, no final de janeiro de 2021, a OMS já registrava 236 pesquisas com candidatas à vacina, estando 63 em fase de ensaios clínicos (World Health Organization, 2021).

3 Entre dezembro de 2020 e janeiro de 2021, resultados de estudos clínicos globais de três vacinas testadas no Brasil foram considerados favoráveis e impulsionaram o início de seu uso em diferentes regiões do mundo. Outras quatro, que não foram testadas no Brasil, começaram a ser aplicadas em outros países em 2020. 
casos confirmados e mais de 120 mil óbitos dos 843.927 somados em todo o mundo. ${ }^{4}$ Esse cenário epidemiológico desastroso - resultante da letalidade do vírus associada às condições históricas de negação de acesso à saúde de grupos particularmente vulnerabilizados, como negros, indígenas e populações periféricas urbanas (Goes; Ramos; Ferreira, 2020; Santos et al., 2020), e ao marcado negacionismo e ineficiência do atual governo (Ortega; Orsini, 2020) - é considerado adequado e necessário pela comunidade científica internacional para a produção rápida e confiável de evidências sobre vacinas contra o coronavírus.

Em vista dessa situação aparentemente paradoxal, na qual se espera que a solução para a pandemia emerja de contextos nos quais ela foi particularmente devastadora, debruço-me sobre matérias jornalísticas, declarações e documentos públicos de autoridades políticas brasileiras para analisar as condições e processos que configuraram o Brasil como um local central da produção global de pesquisas com vacinas contra o coronavírus no primeiro semestre de 2020 . Nesse esforço, problematizo como essa posição foi localmente positivada, a partir do entendimento de autoridades e cientistas de que a procura pelo Brasil como local de pesquisas significava o reconhecimento das competências nacionais no campo científico e uma oportunidade política de o país reverter sua péssima imagem internacional. Ao final, reflito sobre como a participação nos experimentos constituiu uma frágil e incerta estratégia para barganhar uma posição mais favorável na "fila" internacional por acesso às futuras vacinas, fato que implica um contundente problema ético envolvendo a capitalização sobre necropolíticas nacionais no processo de produção de tecnologias de interesse global e as desigualdades internacionais nas condições de acesso a esses bens.

\section{Negação, negacionismo e necropolítica}

No início de janeiro de 2020, a OMS anunciou que um novo coronavírus havia sido identificado em Wuhan, na China, como causa de 41 casos de

4 Ao longo de 2020, o Brasil foi ultrapassado pela Índia no quantitativo de casos de Covid-19, mas manteve-se na segunda posição mundial em óbitos pela doença, ficando atrás apenas dos Estados Unidos. No início de 2021, o Brasil superou a marca de 200 mil óbitos pela doença. Informações atualizadas diariamente no portal da OMS (ver https://covid19.who.int/, acesso em 05/02/2021). 
adoecimentos, com sintomas que incluíam febre, falta de ar e pneumonia. Destes, sete pessoas haviam evoluído para quadros graves e uma faleceu. Naquele momento, a situação chamou a atenção da OMS, pois, embora houvesse sintomas parecidos com os de outras infecções respiratórias, exames laboratoriais haviam descartado prováveis causas virais conhecidas. Segundo os cientistas chineses, estávamos diante de um novo agente infeccioso, cujos maiores índices de perigo estavam em sua alta transmissibilidade, potencial de letalidade e no fato de o organismo humano não lhe ser imune (World Health Organization, 2020b). Em poucas semanas, o vírus demonstrou seus amplos desafios a sistemas econômicos e sanitários, avançando rapidamente sobre diferentes continentes e passando de um agente infeccioso supostamente local para uma ameaça epidemiológica global (Segata, 2020). Em 11 de março, a OMS declarou que estávamos vivendo uma pandemia, com casos confirmados em 114 países (Organização Pan-Americana da Saúde, 2020). Nesse contexto, entendeu-se a busca por uma vacina como imperativa para contenção dos danos humanitários e econômicos associados ao vírus.

O Ministério da Saúde registrou o primeiro caso de Covid-19 no Brasil em 26 de fevereiro de 2020 (Brasil, 2020a). No dia 6 de março, diante da confirmação de mais 12 casos, o presidente Jair Bolsonaro fez seu primeiro pronunciamento oficial sobre o assunto, no qual comunicou que uma série de medidas de vigilância e produção de informação capitaneadas pelo governo federal estavam em curso e afirmou categoricamente que "seguir rigorosamente às recomendações dos especialistas [era] a melhor medida de prevenção". As orientações da OMS incluíam medidas como o distanciamento social ampliado, uso de máscaras e outros equipamentos de proteção individual e higienização contínua das mãos, ações com intensos reflexos na circulação de pessoas e mercadorias, no sistema produtivo e nas práticas de consumo.

As orientações públicas do presidente e o avanço do coronavírus no país se radicalizaram com grande rapidez. No seu terceiro pronunciamento sobre a Covid-19, em 24 de março de 2020, Bolsonaro mudou o tom de suas falas oficiais e passou a produzir mais sistematicamente uma narrativa de negação da gravidade do novo coronavírus. Acusando a mídia de espalhar "pânico" e causar "histeria" na população, e chamando governadores e prefeitos de "irresponsáveis" por adotarem medidas de "confinamento em massa", o presidente chamou a Covid-19 de "gripezinha". Dali em diante, reforçou a proposta de "isolamento vertical", que 
previa que somente um certo "grupo de risco", formado por idosos e pessoas com comorbidades, precisaria tomar medidas de isolamento, devendo a vida cotidiana "voltar à normalidade", de modo a preservar as atividades econômicas. Ao longo dos meses, as práticas de negacionismo incorporaram, ainda, o acionamento de especialistas com posicionamentos divergentes de consensos científicos em formação e a alta seletividade no uso de informações científicas (Camargo Jr.; Coeli, 2020), bem como tentativas de manipulação dos registros de vigilância epidemiológica e exposições públicas de desprezo pelo isolamento social.

Ações e omissões negacionistas provocaram uma profunda desarticulação de políticas de saúde e assistência, cujos resultados foram desigualmente distribuídos, tanto em seu alcance quanto em sua intensidade. Segundo dados do Ministério da Saúde, com registros feitos até 22 de agosto de 2020, somando-se pretos e pardos, temos 46.436 mortes de pessoas negras pela Covid-19, enquanto brancos totalizam 33.531 mortes (Brasil, 2020b). ${ }^{5}$ Informações epidemiológicas do mesmo órgão apontam, ainda, que até a mesma data já havia casos confirmados em todos os Distritos Sanitários Especiais Indígenas (DSEI), totalizando 21.310 indígenas infectados, dos quais 353 foram a óbito (Brasil, 2020d). A disparidade racial mostra-se, entretanto, mais flagrante quando observados dados indiretos do impacto da pandemia sobre brancos e negros. Dados de registros cartoriais de óbito disponíveis no Portal da Transparência apontam que entre os meses de março e junho de 2020 as mortes por causas respiratórias aumentaram $34,5 \%$ se comparadas ao mesmo período de 2019, sendo que "o registro de mortes por doenças respiratórias entre aqueles declarados pretos e pardos cresceu mais de $70 \%$ durante os meses da pandemia", enquanto o aumento foi de cerca de $25 \%$ entre brancos (Mortes..., 2020).

Análises sobre as razões para que a Covid-19 venha se mostrando mais letal para negros e negras apontam não só atitudes negacionistas do governo, mas, principalmente, os impactos do racismo na saúde (Goes; Ramos; Ferreira, 2020; Santos et al., 2020). Políticas históricas de governo reiteram para esses grupos as piores condições de habitação e transporte, acesso a serviços e tecnologias de saúde e trabalho digno, afastando-lhes de condições mínimas de adesão a

5 Entre povos indígenas, nota-se também uma significativa desigualdade. Enquanto, no mês de junho, a taxa letalidade nacional estava em $5,2 \%$, entre indígenas o percentual era de $8,8 \%$ (Articulação dos Povos Indígenas do Brasil, 2020). 
procedimentos profiláticos; ao tempo em que são negros os sujeitos mais acometidos de comorbidades que agravam a evolução da Covid-19 (Ribeiro; Lima; Waldman, 2020). Nesse sentido, a dinâmica brasileira da pandemia não encontra explicações em um recorte temporal discreto, estritamente coincidente com os desenhos das curvas epidemiológicas iniciadas com a pandemia. Ao contrário, seu desenvolvimento está relacionado a temporalidades profundas, nas quais o negacionismo contemporâneo atualiza e diversifica efeitos devastadores de negações históricas de direitos relacionados à saúde de populações negras (Carneiro, 2005). ${ }^{6}$

O contexto da Covid-19 no Brasil, portanto, contradiz narrativas divulgadas no início de 2020, nas quais se descrevia o coronavírus como um agente "democrático", que desconheceria identidades raciais ou classes sociais. As estimativas numéricas, que sinalizam para a concretude de trajetórias precarizadas de acesso a equipamentos básicos à vida digna, indicam que os óbitos em maior proporção de negros e negras na pandemia se configuram mediante operações necropolíticas (Mbembe, 2016), nas quais dispositivos e tecnologias de governo interagem reiterada e seletivamente na efetivação de mortes acentuadas desses sujeitos. Nesse contexto, os modos de gestão do avanço da pandemia tanto revelam quanto atualizam dinâmicas de estruturação do racismo, reconhecível nos processos (des)organizacionais mediante os quais se rearticulam acondicionamentos evitáveis de sujeitos e grupos negros em posições e situações que lhes expõem desproporcionalmente ao adoecimento e à morte.

\section{Experimentos vacinais e conversões necroeconômicas}

Os posicionamentos públicos do presidente Bolsonaro, os resultados da ausência de políticas nacionais de controle da pandemia e a sabotagem de

6 Em sua página na internet, a frente do movimento indígena no enfrentamento da pandemia afirma que a vulnerabilização de grupos indígenas à Covid-19 está relacionada tanto à histórica política colonial-estatal de genocídio quanto às suas atualizações no contexto da pandemia sobretudo aquelas relacionadas ao avanço de atividades ilegais em terras indígenas: "As invasões aos territórios estão mais intensas, o desmatamento aumentou, missionários fundamentalistas continuam sendo incentivados a cometer crimes e a mineração ilegal avança dentro das terras indígenas" (Dados..., [2021]). 
estratégias estaduais e municipais de isolamento social foram seriamente criticados na mídia internacional. Dentre inúmeras matérias com esse teor, a que provavelmente teve maior destaque foi o editorial do jornal The Washington Post (Leaders..., 2020), publicado em 14 de abril de 2020. Ao avaliar que "a severidade da epidemia em muitas nações tem dependido de quão bem ou mal os governantes responderam a ela", o editorial afirma categoricamente que "de longe, o caso mais sério de má conduta é o do presidente brasileiro Jair Bolsonaro" (Leaders..., 2020, tradução minha). À medida que os indicadores da pandemia no Brasil pioravam rapidamente, as abordagens desse e de outros veículos se tornaram ainda mais mordazes, enunciando uma corrosão da imagem internacional do país. Um estudo sobre as percepções acerca do Brasil no exterior, realizado a partir de publicações de periódicos de impacto internacional, avaliou que "a imagem do governo Bolsonaro no exterior é de crise ética e falência da gestão" e que "o tema Covid-19 foi responsável por 68\% do total da cobertura negativa no segundo trimestre" (Curado; Couto; Gutierrez, 2020).?

A negatividade da imagem brasileira foi acompanhada de reportagens internacionais e nacionais que requalificaram suas taxas de morbidade e mortalidade por Covid-19 como um elemento potencialmente positivo no combate à pandemia. Ao registrarem o avanço de pesquisas com possíveis vacinas contra o coronavírus e o afluxo de ensaios clínicos para o Brasil, algumas matérias destacaram o fato ambíguo de o quadro epidemiológico brasileiro ter sido identificado pela comunidade científica e empresas do setor como aquele que oferecia condições à rápida conclusão das pesquisas e ao vislumbre do lançamento de uma solução imunizante. Passaram então a emergir na mídia certos discursos que descreviam o país como local que "oferece esperança para o resto mundo" (Walsh et al., 2020), ao mesmo tempo que, internamente, veículos de imprensa enquadravam a escolha do Brasil por laboratórios estrangeiros como uma possibilidade de reversão de sua imagem internacional. Entendeu-se, nesse sentido, a participação do Brasil nos experimentos como uma "oportunidade histórica", mediante a qual "podemos nos redimir de nossa atuação desastrosa frente à pandemia, nos tornando uma liderança global em busca da vacina para o grande mal do século XXI" (Piccolotto, 2020).

7 A amostra do estudo foi formada por matérias dos periódicos The New York Times, The Washington Post, The Guardian, The Economist, El País, Le Monde e Der Spiegel. 
O entusiasmo com a participação brasileira em pesquisas deveu-se à quantidade crescente de estudos com vacinas que o país passou a hospedar na primeira metade de 2020. A aprovação do primeiro ensaio clínico internacional no Brasil ocorreu em junho daquele ano, tendo a candidata desenvolvida pela Universidade de Oxford e a farmacêutica AstraZeneca (chamada de "vacina de Oxford") recebido anuência da Agência Nacional de Vigilância Sanitária (Anvisa) e da Comissão Nacional de Ética em Pesquisa (Conep) para ser inoculada em milhares de brasileiros, de modo a verificar sua eficácia imunogênica (Agência Nacional de Vigilância Sanitária, 2020a; Brasil, 2020). De junho a agosto de 2020, essas duas instâncias regulatórias aprovaram mais três estudos clínicos, estabelecendo regimes prioritários de avaliação e autorizações em tempo recorde (Comissão..., 2020). Desde então, estão em curso quatro experimentos com vacinas (Quadro 1), cujos protocolos preveem o recrutamento de mais de 33 mil sujeitos de pesquisa no país. ${ }^{8}$

\begin{tabular}{|c|c|c|}
\hline Laboratórios produtores & $\begin{array}{c}\text { Previsão de recrutamento } \\
\text { no Brasil }\end{array}$ & $\begin{array}{c}\text { Transferência de } \\
\text { tecnologia associada }\end{array}$ \\
\hline $\begin{array}{c}\text { Universidade de Oxford } \\
\text { e AstraZeneca }\end{array}$ & 10.000 & Sim (Fiocruz) \\
\hline $\begin{array}{c}\text { Sinovac Biotech } \\
\text { e Instituto Butantan }\end{array}$ & 13.060 & Sim (Instituto Butantan) \\
\hline Pfizer e BioNTech & 3.100 & Não \\
\hline Janssen-Cilag & 7.560 & Não \\
\hline
\end{tabular}

Quadro 1. Ensaios clínicos internacionais com vacinas aprovados no Brasil. Fonte: elaboração própria, com informações da Anvisa, atualizadas em 18 de janeiro de 2021 (Agência Nacional de Vigilância Sanitária, 2021).

8 Em meados de 2020, a previsão de recrutamento no Brasil era de aproximadamente $22 \mathrm{mil}$ pessoas (Agência Nacional de Vigilância Sanitária 2020b; Brasil, 2020c). Ao longo do segundo semestre de 2020 , todos os estudos aqui realizados ampliaram o quantitativo inicialmente projetado de inclusão de participantes de pesquisa. 
Os testes em humanos constituem pontos de passagem obrigatória (Callon, 1984) dos protocolos internacionais para produção e desenvolvimento de vacinas. De modo geral, os estudos clínicos mais avançados, como os que estão em curso no Brasil, seguem o método randomizado internacional duplo-cego controlado, cuja necessidade de altas quantidades de sujeitos em situações de risco de saúde articula as práticas e interesses de cientistas, gestores públicos e laboratórios privados a contextos sanitários precarizados do sul global (Castro, 2020a; Petryna, 2009). No contexto da pandemia de Covid-19, as condições para realização desses experimentos incluem, ainda, fatores como equipes de pesquisadores capacitados para manejar o produto experimental e analisar seus efeitos clínicos, investidores com interesse e capacidade suficientes para assumir o risco de os resultados serem insatisfatórios, laboratórios com capacidade de produção de milhões de doses para os testes e milhares sujeitos dispostos a tomar injeções com as candidatas. ${ }^{9}$

Nenhum desses fatores, entretanto, é contornável diante da primazia da necessidade de submissão das candidatas à vacina a um teste de risco. Conforme explicado em entrevista ao podcast Café da Manhã por Esper Kallás (2020), coordenador nacional da pesquisa com a candidata da empresa Sinovac, o que destacou o Brasil na atração de estudos clínicos foi o fato de aqui haver uma ampla dispersão do vírus na população no momento dos testes, quadro contrastante com a situação de epicentros anteriores da pandemia. Locais como a China e a Itália, que concentraram a maior quantidade de casos de Covid-19 no mundo nos primeiros meses de 2020, encontravam-se com curvas baixas de contágio no segundo trimestre daquele ano, situação que direcionou as pesquisas mais avançadas justamente para onde as políticas de contenção do alastramento do coronavírus fracassaram.

E quando você quer provar que uma vacina vai proteger as pessoas, o local ideal é onde está tendo mais disseminação. [...] Se você vai fazer esse mesmo estudo hoje em Milão, não está tendo mais transmissão. Se você vai fazer esse estudo

9 Outro elemento que se mostrou fundamental para o trânsito de pesquisas para o Brasil foi o reconhecimento da população brasileira como racial e etnicamente "diversa" e, por isso, interessante para a qualificação e extrapolação dos resultados dos ensaios clínicos (Castro, 2020b). 
hoje em Wuhan, na China, praticamente não está tendo transmissão. [...] Então, é essa a razão que trouxe isso pra cá. (Kallás, 2020).

A chegada de experimentos com vacinas ao Brasil foi celebrada por diferentes cientistas e autoridades públicas, como um sinalizador do reconhecimento internacional da capacidade científica, econômica e política brasileira de atrair investimentos e de aproveitar oportunidades para estabelecer acordos de capacitação e transferência de tecnologia. Caracterizações nesse sentido foram marcantes nos discursos de gestores e pesquisadores envolvidos no estudo da "vacina de Oxford", então considerada pela OMS a mais "avançada" dentre as que se encontram em fases de testes (Nebehay, 2020). Segundo declarado em entrevista à revista Veja por Sue Ann Clemens, cientista que coordenou as tratativas com Oxford para o trânsito dessa pesquisa para o país, a realização de estudos com vacinas "mostra para o mundo que as nossas agências regulatórias têm agilidade e qualidade para fazer uma revisão de alto nível em um curto espaço de tempo e para a equipe envolvida na condução desses estudos, significa capacitação tanto na realização de testes clínicos de grande porte quanto no desenvolvimento de vacinas" (Vidale, 2020).

Considerações semelhantes foram feitas, inclusive, por cientistas que se posicionaram de modo contundentemente crítico com relação às ações e omissões do governo federal no combate à pandemia. Nesse sentido, destaca-se 0 posicionamento de Natália Pasternak, presidente do Instituto Questão de Ciência e importante referência no debate público nacional contra o negacionismo científico. Em entrevista ao portal BR Político, ela afirmou que era justamente o fato de o país se configurar genericamente como um ambiente de risco o que lhe colocou na linha de frente do desenvolvimento global de vacinas, explicitando uma ambígua relação entre o afluxo dos experimentos com candidatas a vacinas e os efeitos da ausência de políticas eficientes de combate à Covid-19 no Brasil.

Essa é a vantagem que a nossa incompetência pode oferecer para esse esforço internacional. Como somos muito incompetentes em conter a doença, nós oferecemos um cenário favorável para a testagem de vacinas. A gente tem o vírus circulando por aqui, enquanto outros países já não têm. E você precisa dessa circulação para expor as pessoas já vacinadas e aquelas que receberam o placebo 
a uma situação de risco de contrair a doença. O Brasil oferece esse risco, principalmente aos profissionais de saúde. Pelo menos essa contribuição a gente pode dar, o que é muito irônico. (Miranda, 2020).

A realização de pesquisas clínicas no país denotou, assim, uma rápida conversão do trágico quadro sanitário nacional em um contexto de "transmissão ativa" ou de "alta circulação do vírus", necessários aos estudos clínicos, bem como deste em oportunidades científicas e políticas para participação em iniciativas internacionais. As trajetórias terapêuticas precarizadas e as vidas perdidas, bem como as necessárias políticas de saúde e assistência para que grupos vulnerabilizados pudessem melhor enfrentar a pandemia, perderam ênfase diante das redescrições operacionalizadas para a condução célere das pesquisas e das apostas em uma futura vacina. Essa transmutação das precariedades em oportunidades, bem como as estratégias de saúde centradas em intervenções tecnológicas, reforçam predicados centrais da inserção do Brasil no mercado global da experimentação farmacêutica, mediante conversões de doenças com alta prevalência em "áreas terapêuticas" passíveis de serem exploradas por meio da incorporação do país em planos internacionais de pesquisa (Castro, 2020a).

Autoridades públicas do campo da saúde também identificaram no afluxo de estudos para o país um reconhecimento internacional de suas capacidades de participar do campo da pesquisa clínica global. Quando da aprovação do quarto ensaio clínico no Brasil, Gustavo Mendes, gerente-geral de medicamentos da Anvisa, explicou em um vídeo institucional que a vinda de mais um experimento não era explicada apenas pelo fato de haver "vírus circulando na população", mas também pelo reconhecimento dado ao país para realização desse tipo de pesquisa, tanto científica quanto regulatoriamente:

[...] existe um reconhecimento do país na capacidade de gerar resultados confiáveis de estudos clínicos. Isso é um reconhecimento não só da agência reguladora, mas também dos centros de pesquisa e dos pesquisadores que estão trabalhando diretamente no desenvolvimento. (Quarto..., 2020).

Nesse contexto, condições crônicas e agudas de saúde vivenciadas por sujeitos brasileiros, desigualmente vetorizadas pelo racismo, são mercantilizadas e capitalizadas por empreendedores, gestores e cientistas locais no mercado 
global farmacêutico, configurando-se em ativos necroeconômicos incorporados ao processo de produção biotecnológica. Destaco, nesse sentido, o contraste entre as ineficientes ou inexistentes estratégias federais de contenção de contágios e mortes e seus esforços para atração e retenção de estudos clínicos com vacinas por meio da expedição de autorizações para pesquisas em curtíssimos prazos, articulação essa que torna a população simultaneamente mais vulnerável à pandemia e disponível aos experimentos farmacêuticos. A pandemia, nesse contexto, tem servido à rearticulação do Brasil no cenário internacional como local que consegue capitalizar sobre seus trágicos e atraentes quadros epidemiológicos, disponibilizando-os às demandas internacionais de produção biotecnológica e de "desburocratização" da arena de negócios.

\section{Reflexões finais: nacionalismo vacinal e desafios éticos}

Além de oportunidades científicas e políticas, os testes clínicos no Brasil têm sido traduzidos em limitadas estratégias de barganha por uma posição mais favorável na "fila" pelas primeiras doses de vacinas. Dentre as quatro candidatas atualmente testadas no país, duas contam com acordos entre governos federal e do estado de São Paulo e os laboratórios fabricantes para acesso aos produtos dos estudos. ${ }^{10}$ Para a vacina produzida pela Sinovac, o governo de São Paulo estabeleceu um acordo de transferência de tecnologia, que permitirá ao Instituto Butantan produzir doses de vacinas a serem publicamente distribuídas. De modo semelhante, o governo federal negociou um acordo com a AstraZeneca que permitirá a formulação, o envase e o controle de qualidade de produção da "vacina de Oxford" pela Fundação Oswaldo Cruz. As expectativas das autoridades brasileiras eram de que, uma vez demonstradas eficácia e segurança satisfatórias para tais produtos, o país tivesse autonomia imediata para produzir doses de vacinas para distribuição e imunização nacionais.

10 No dia 17 de janeiro de 2021, a Anvisa concedeu autorização para uso emergencial no Brasil das candidatas à vacina da Universidade de Oxford/AstraZeneca e da Sinovac Biotech. Segundo a agência, trata-se de uma permissão temporária e excepcional, voltada à "utilização durante a atual emergência em saúde pública, de modo a minimizar, o mais rápido possível, os impactos da crise" (Anvisa..., 2021). 
Tais acordos foram estabelecidos em um contexto no qual a autonomia produtiva local mostrava-se especialmente crítica, sobretudo diante de políticas nacionalistas que caracterizaram as ações de governantes de países mais ricos (Makovicky, 2020). Mesmo sem testes conclusivos, algumas farmacêuticas estabeleceram acordos com os governos dos Estados Unidos, Reino Unido, Japão e países da União Europeia, dentre outros, para aquisição antecipada de milhões de doses das vacinas (Moreira, 2020). Nesse cenário, no qual a corrida dos experimentos foi acompanhada de uma competição para fechar acordos antecipados, o diretor-geral da OMS, Tedros Adhanom, demonstrou preocupações com o "nacionalismo vacinal" (WHO chief..., 2020), situação na qual autoridades de países ricos reservam doses de vacinas para imunização prioritária de suas populações. Essa situação daria seguimento a práticas que ocorreram nos primeiros meses da pandemia, nos quais países como Estados Unidos e França compraram, estocaram e confiscaram enormes quantidades de equipamentos de proteção individual, respiradores e reagentes de testes diagnósticos e, assim, dificultaram o acesso de países como o Brasil a itens de extrema necessidade (Castro, 2020c).

Com o previsível desequilíbrio entre oferta e demanda quando do lançamento das primeiras vacinas, a OMS sinalizou que países do sul global poderiam sofrer ainda mais com o prolongamento dos efeitos da pandemia, ao tempo que países ricos consumissem rapidamente as primeiras unidades das vacinas. Com o avanço das estratégias de "nacionalismo vacinal", as perspectivas, eram de que "as vacinas chegarão eventualmente à maioria das populações, mas somente após países poderosos terem se protegido" (Fidler, 2020, p. 749, tradução minha). Países como o Brasil, mesmo tendo participação fundamental nos experimentos e cujos milhares de habitantes assumiram riscos viscerais do desenvolvimento dessas tecnologias, provavelmente precisariam buscar acordos bilaterais, pois, até meados de 2020 , as garantias de acesso não estavam necessariamente baseadas nos quadros epidemiológicos ou na dimensão dos riscos experimentais assumidos, mas nos recursos disponíveis para comprar as vacinas diretamente dos fabricantes.

Tal cenário veio a se confirmar com o lançamento dos resultados dos testes clínicos de algumas candidatas à vacina. Reino Unido, União Europeia e os Estados Unidos foram alguns dos primeiros cujas autoridades sanitárias aprovaram o uso emergencial de vacinas e, ainda em 2020, seus respectivos 
governos começaram a acionar o amplo estoque de doses que haviam adquirido. ${ }^{11}$ Nesse contexto, apesar de o Brasil ter se constituído como uma "linha de frente" das pesquisas clínicas, ficamos para trás na corrida do acesso às vacinas, com doses insuficientes até para imunização de profissionais de saúde no início de 2021. Destacam-se como componentes dessa situação as limitações dos acordos de transferência tecnológica assumidos com laboratórios estrangeiros; a necessidade de insumos importados e altamente disputados para produção local e a inação deliberada do governo federal para estabelecer contratos de compra com diferentes laboratórios. Ressalto, ainda, o desestímulo à vacinação dado pelo presidente Bolsonaro, que tem enfatizado a vacinação como questão de escolha individual e ventilado teses conspiratórias sobre efeitos adversos associados às vacinas. ${ }^{12}$ Nesse contexto, os grupos de sujeitos mais vulnerabilizados pela pandemia ficam expostos de modo prolongado a contágios e risco de morte evitáveis, enquanto políticas diversas dificultam seu acesso às tecnologias cujo desenvolvimento e distribuição se instituem continuamente sob a "dialética da inovação e da inequidade" (Rusert, 2019, p. 29).

$\mathrm{O}$ avanço das etapas da corrida tecnológica não significou o recuo imediato da pandemia, mas abriu novos capítulos da trágica articulação entre inequidades globais, históricos nacionais de negação de direitos à saúde e políticas negacionistas na configuração da pandemia no Brasil. Os processos de produção, experimentação, comercialização e distribuição de tecnologias médicas nesse contexto denotam, assim, que as desigualdades entre países do norte e do sul global, bem como as inequidades de saúde entre brancos e negros no Brasil, não só configuram diferentes temporalidades e intensidades da pandemia,

11 Enquanto o Canadá, por exemplo, comprou cinco vezes mais doses do que o necessário para imunizar toda a sua população (Coronavirus..., 2021, p. 2), a maior parte dos países do sul global não têm perspectivas de quando terão vacinas suficientes para seus profissionais de saúde e idosos. Em um pronunciamento realizado em 18 de janeiro de 2021, o diretor-geral da OMS afirmou que até aquele momento mais de 39 milhões de doses da vacina já haviam sido administradas em pelo menos 49 países de alta renda; já na Guiné-Equatorial, um país de baixa renda, apenas 25 doses tinham sido distribuídas (Chefe..., 2021).

12 Refiro-me, por exemplo, à declaração pública de Bolsonaro feita em dezembro de 2020, na qual insinuou que a vacina da empresa Pfizer poderia alterar o código genético de humanos e transformá-los em jacarés. Tais declarações enfraquecem o debate público sobre a importância da vacinação e a responsabilidade do governo em promover o amplo acesso da população às vacinas. 
mas são elementos centrais do desenvolvimento e do acesso às vacinas nas quais se aposta o fim do "mal do século". Por outro lado, se desigualdades não forem efetivamente combatidas, o advento de uma vacina não encerrará os males da pandemia para todos, nem com a mesma rapidez e efetividade. A experimentação de vacinas no Brasil denota como os mecanismos de exploração de grupos populacionais vulnerabilizados em pesquisas médicas sucedem mediante a captura dos processos de experimentação pela lógica do capital (Sunder Rajan, 2007), nos quais desumanizações e mortes vetorizadas pelo racismo tornam-se ativos necroeconômicos do mercado biotecnológico, e os produtos desses dispositivos tendem a ser menos acessíveis àqueles que, de modo mais substancial, vivenciam os riscos envolvidos no seu desenvolvimento.

\section{Referências}

AGÊNCIA NACIONAL DE VIGILÂNCIA SANITÁRIA. Autorizado estudo clínico de potencial vacina contra Covid-19. Brasília: Anvisa, 3 jun. 2020a. Disponível em: https:// www.gov.br/anvisa/pt-br/assuntos/noticias-anvisa/2020/autorizado-estudo-clinico-de-potencial-vacina-contra-covid-19. Acesso em: 29 jan. 2021.

AGÊNCIA NACIONAL DE VIGILÂNCIA SANITÁRIA. Covid-19: Anvisa autoriza novo ensaio clínico de vacina. Brasília: Anvisa, 18 ago. 2020b. Disponível em: https://www. gov.br/anvisa/pt-br/assuntos/noticias-anvisa/2020/covid-19-anvisa-autoriza-novo-ensaio-clinico-de-vacina Acesso em: 29 jan. 2021.

AGÊNCIA NACIONAL DE VIGILÂNCIA SANITÁRIA. Covid-19: fique por dentro do mapa das vacinas em teste no Brasil. Brasília: Anvisa, 18 jan. 2021. Disponível em: https://www.gov.br/anvisa/pt-br/assuntos/noticias-anvisa/2020/fique-por-dentro-do-mapa-das-vacinas-em-teste-no-brasil. Acesso em: 29 jan. 2021.

ANDREONI, M.; LONDOÑO, E. Coronavirus crisis has made Brazil an ideal vaccine laboratory. The New York Times, New York, 15 Aug. 2020. Disponível em: https://www. nytimes.com/2020/08/15/world/americas/brazil-coronavirus-vaccine.html. Acesso em: 20 ago. 2020.

ANVISA aprova por unanimidade uso emergencial das vacinas. In: GOV.BR. Brasília: Governo do Brasil, 17 jan. 2021. Disponível em: https://www.gov.br/pt-br/noticias/ saude-e-vigilancia-sanitaria/2021/01/anvisa-aprova-por-unanimidade-uso-emergencial-das-vacinas. Acesso em: 29 jan. 2021. 
ARTICULAÇÃO DOS POVOS INDÍGENAS DO BRASIL. Boletim semanal da APIB sobre o novo coronavírus entre os povos indígenas $n^{\circ}$ 04. [S. l.]: Apib, 2020.

BRASIL. Ministério da Saúde. Boletim Epidemiológico 06. Brasília: MS, 3 abr. 2020a. Disponível em: https://portalarquivos.saude.gov.br/images/pdf/2020/April/03/BE6-Boletim-Especial-do-COE.pdf. Acesso em: 28 ago. 2020.

BRASIL, Ministério da Saúde. Boletim epidemiológico especial $n^{\circ} 28$. Brasília: MS, 26 ago. 2020b. Disponível em: https://antigo.saude.gov.br/images/pdf/2020/August/27/ Boletim-epidemiologico-COVID-28-FINAL-COE.pdf. Acesso em: 28 ago. 2020.

BRASIL. Ministério da Saúde. Conselho Nacional de Saúde. Comissão Nacional de Ética em Pesquisa. Boletim Ética em Pesquisa. Edição Especial Coronavírus (Covid-19). Edição 50. Brasília: CONEP/CNS/MS, 2020c. Disponível em: http://conselho.saude. gov.br/images/comissoes/conep/img/boletins/Boletim-EticaemPesquisa-ED50.pdf. Acesso em: 28 ago. 2020.

BRASIL. Ministério da Saúde. Informe epidemiológico $n^{0}$ 16. Doença Coronavírus (COVID-19) em populações indígenas. Brasília: MS, 2020d. Disponível em: https:// saudeindigenal.websiteseguro.com/coronavirus/pdf/Informe\%20Epidemiologico\%20SE\%2034\%20-\%20SESAI\%20COVID\%2019.pdf. Acesso em: 3 fev. 2021.

CALLON, M. Some elements of a sociology of translation: domestication of scallops and the fishermen of St. Brieuc Bay. Sociological Review, London, v. 32, n. 1, p. 196-233, 1984.

CAMARGO JR., K.; COELI, R. A difícil tarefa de informar em meio a uma pandemia. Physis, Rio de Janeiro, v. 30, n. 2, e300203, 2020.

CARNEIRO, S. A construção do Outro como Não-Ser como fundamento do Ser. 2005. Tese (Doutorado em Educação) - Faculdade de Educação, Universidade de São Paulo, São Paulo, 2005.

CASTRO, R. Economias políticas da doença e da saúde: uma etnografia da experimentação farmacêutica. São Paulo: Hucitec, 2020a.

CASTRO, R. Covid-19 e economias da diversidade: uma crítica antropológica da biologização da raça nos ensaios clínicos com vacinas. ClimaCom, ano 7, n. 19, 2020b. Disponível em: http://climacom.mudancasclimaticas.net.br/covid-19-e-diversidade/. Acesso em: 22 dez. 2020.

CASTRO, R. Ciências e tecnologias na pandemia de Covid-19: exposições, experimentos, expectativas. In: GROSSI, M.; TONIOL, R. (org.). Cientistas sociais e o coronavírus. São Paulo: Anpocs; Florianópolis: Tribo da Ilha, 2020c. p. 359-362. 
CHEFE da OMS alerta para um "fracasso moral" da vacinação contra COVID-19. In: NAÇÕES UNIDAS BRASIL. Brasília: Casa ONU Brasil, 19 jan. 2021. Disponível em: https://brasil.un.org/pt-br/108239-chefe-da-oms-alerta-para-um-fracasso-moral-da-vacinacao-contra-covid-19. Acesso em: 3 fev. 2021.

COMISSÃO do CNS aprova terceira fase dos testes da vacina contra Covid-19. In: CONSELHO NACIONAL DE SAÚDE. Brasília: CNS, 10 jul. 2020. Disponível em: http://conselho.saude.gov.br/ultimas-noticias-cns/1263-comissao-do-cns-aprova-terceira-fase-dos-testes-da-vacina-contra-covid-19. Acesso em: 20 ago. 2020.

CORONAVIRUS vaccines: expect delays. The Economist Intelligence Unit, [s. l.], 27 Jan. 2021. Disponível em: https://www.eiu.com/n/campaigns/q1-global-forecast-2021/?utm_source=twitter\&utm_medium=organic_social\&utm_ campaign=q1_2021_global_forecast\&utm_term=stark_divide\&utm_content=map\& linkId=100000030033676. Acesso em: 4 fev. 2021.

CURADO, O.; COUTO, E.; GUTIERREZ, J. Estudo Brasil imagem internacional $2^{\circ}$ trimestre 2020. [S. l.]: Curado \& Associados, 2020.

DADOS Covid-19. In: EMERGÊNCIA INDÍGENA. [S. l.]: Apib: Aty Guasu: CGY: Coiab: Arpin Sudeste: Apoinme: Arpin Sul: Conselho Povo Terena, [2021]. Disponível em: https://emergenciaindigena.apiboficial.org/dados_covid19/. Acesso em: 3 fev. 2021.

FIDLER, D. Vaccine nationalism's politics. Science, New York, v. 369, n. 6505, p. 749, 2020.

GOES, E. F.; RAMOS, D. O.; FERREIRA, A. J. F. Desigualdades raciais em saúde e a pandemia da Covid-19. Trabalho, Educação e Saúde, Rio de Janeiro, v. 18, n. 3, e00278110, 2020.

KALLÁS, E. Por que quem produz vacinas da Covid-19 está de olho no Brasil. Entrevista a Rodrigo Viseu e Magê Flores para o podcast Café da Manhã, São Paulo, 24 jun. 2020. Disponível em: https://wwwl.folha.uol.com.br/podcasts/2020/06/por-que-quem-produz-vacinas-da-covid-19-esta-de-olho-no-brasil-ouca-podcast.shtml. Acesso em: 31 ago. 2020.

LEADERS risk lives by minimizing the coronavirus. Bolsonaro is the worst. The Washington Post, Washington, 14 Apr. 2020. Disponível em: https://www. washingtonpost.com/opinions/global-opinions/jair-bolsonaro-risks-lives-by-minimizing-the-coronavirus-pandemic/2020/04/13/6356a9be-7da6-11ea-9040-68981f488eed_story.html. Acesso em: 19 ago. 2020.

MAKOVICKY, N. The national(ist) necropolitics of masks. Social Anthropology, Hoboken, v. 28, n. 2, p. 341-315, 2020.

MBEMBE, A. Necropolítica. Arte \& Ensaios, Rio de Janeiro, n. 32, p. 123-151, 2016. 
MIRANDA, C. 'Incompetência' do Brasil em conter o vírus cria cenário 'favorável' para vacinas, diz especialista. BRPolítico, São Paulo, 5 jul. 2020. Disponível em: https://brpolitico.com.br/noticias/incompetencia-do-brasil-em-conter-o-virus-cria-cenario-favoravel-para-vacinas-diz-especialista/. Acesso em: 3 fev. 2021.

MOREIRA, A. Brasil precisa decidir sobre como terá acesso a vacina contra covid19. Valor Investe, Rio de Janeiro, 20 ago. 2020. Disponível em: https://valorinveste. globo.com/mercados/brasil-e-politica/noticia/2020/08/20/brasil-precisa-decidir-sobre-como-tera-acesso-a-vacina-contra-covid-19.ghtml. Acesso em: 25 ago. 2020.

MORTES entre pretos e pardos por doenças respiratórias crescem mais do que entre brancos durante a pandemia, segundo cartórios. G1, Rio de Janeiro, 13 jul. 2020. Disponível em: https://gl.globo.com/bemestar/coronavirus/noticia/2020/07/13/mortes-de-pretos-e-pardos-tiveram-alta-de-31percent-na-pandemia-segundo-cartorios. ghtml. Acesso em: 31 ago. 2020.

NEBEHAY, S. Candidata a vacina da AstraZeneca é a mais avançada contra Covid-19, diz OMS. Reuters, [S. l.], 26 jun. 2020. Disponível em: https://br.reuters.com/article/ topNews/idBRKBN23X217-OBRTP. Acesso em: 25 ago. 2020.

ORGANIZAÇÃO PAN-AMERICANA DA SAÚDE. OMS afirma que Covid-19 é agora caracterizada como pandemia. Brasília: Opas Brasil, 11 mar. 2020. Disponível em: https:// www.paho.org/bra/index.php?option=com_content\&view=article\&id=6120:oms-afirma-que-covid-19-e-agora-caracterizada-como-pandemia\&Itemid=812. Acesso em: 28 ago. 2020.

ORTEGA, F.; ORSINI, M. Governing Covid-19 without government in Brazil: ignorance, neoliberal authoritarianism, and the collapse of public health leadership. Global Public Health, Abingdon, v. 15, n. 9, p. 1257-1277, 2020.

PETRYNA, A. When experiments travel: clinical trials and the global search for human subjects. Princeton: Princeton University Press, 2009.

PICCOLOTTO, L. Brasil pode mudar de mau exemplo a líder global pela vacina contra Covid-19. UOL, São Paulo, 10 ago. 2020. Disponível em: https://govtech.blogosfera. uol.com.br/2020/08/10/uma-otima-noticia-brasil-pode-ser-lideranca-em-vacina-contra-covid-19/. Acesso em: 20 ago. 2020.

QUARTO estudo de vacina aprovado. Brasília: Anvisa, 18 ago. 2020. 1 vídeo (3min21s). Publicado no canal Agência Nacional de Vigilância Sanitária. Disponível em: https:// www.youtube.com/watch?v=1fVfjF69_t0\&feature=emb_title. Acesso em: 4 fev. 2021.

RIBEIRO, H.; LIMA, V. M.; WALDMAN, E. A. In the Covid-19 pandemic in Brazil, do brown lives matter?. The Lancet Global Health, [s. l.], v. 8, p. e976-e977, 2020. 
RUSERT, B. Naturalizing coercion: the Tuskegee experiments and the laboratory life of the plantation. In: BENJAMIN, R. (ed.). Captivating technology: race, carceral technoscience, and liberatory imagination in everyday life. Durham: Duke University Press, 2019. p. 25-49.

SANTOS, M. et al. População negra e Covid-19: reflexões sobre racismo e saúde. Estudos Avançados, São Paulo, v. 34, n. 99, p. 225-243, 2020.

SEGATA, J. Covid-19, biossegurança e antropologia. Horizontes Antropológicos, Porto Alegre, ano 26, n. 57, p. 275-313, maio/ago. 2020.

SUNDER RAJAN, K. Experimental values - Indian clinical trials and surplus health. New Left Review, London, v. 45, p. 67-88, 2007.

VIDALE, G. ‘O Brasil se tornou protagonista', diz pesquisadora da vacina de Oxford. Veja, São Paulo, 29 jul. 2020. Disponível em: https://veja.abril.com.br/saude/o-brasil-se-tornou-protagonista-diz-pesquisadora-da-vacina-de-oxford/. Acesso em: 20 ago. 2020.

WALSH, N. et al. The world's hopes for a coronavirus vaccine may run in these health care workers' veins. CNN, [s. l.], 27 July 2020. Disponível em: https://edition.cnn. com/2020/07/27/americas/brazil-covid-19-sinovac-vaccine-intl/index.html. Acesso em: 20 ago. 2020.

WHO CHIEF warns against COVID-19 'vaccine nationalism', urges support for fair access. UN News, [s. l.], 18 Aug. 2020. Disponível em: https://news.un.org/en/ story/2020/08/1070422. Acesso em: 25 ago. 2020.

WORLD HEALTH ORGANIZATION. Draft landscape of Covid-19 candidate vaccines. [S. l.]: WHO, 2020a. Disponível em: https://www.who.int/publications/m/item/draft-landscape-of-covid-19-candidate-vaccines. Acesso em: 31 ago. 2020.

WORLD HEALTH ORGANIZATION. Novel coronavirus - China. [S. l.]: WHO, 12 Jan. 2020b. Disponível em: https://www.who.int/csr/don/12-january-2020-novel-coronavirus-china/en/. Acesso em: 28 ago. 2020.

WORLD HEALTH ORGANIZATION. COVID-19 - Landscape of novel coronavirus candidate vaccine development worldwide. [S. l.]: WHO, 2021. Disponível em: https://www. who.int/docs/default-source/coronaviruse/novel-coronavirus_landscape_covid1942359771-d8bb-4f89-8b72-5b02b0d69acf.zip?sfvrsn=617c8d90_16\&download=true. Acesso em: 28 jan. 2021.

Recebido: 31/08/2020 Aceito:20/01/2021 | Received:8/31/2020 Accepted:1/20/2021 